\title{
RADIO OBSERVATIONS OF INTERPLANETARY MAGNETIC FIELD STRUCTURES OUT OF THE ECLIPTIC
}

\author{
R. J. FITZENREITER, J. FAINBERG, and R. R. WEBER \\ NASA/Goddard Space Flight Center, \\ Laboratory for Extraterrestrial Physics, Greenbelt, Md. 20771, U.S.A. \\ and \\ H. ALVAREZ, ${ }^{*}$ F. T. HADDOCK, and W. H. POTTER \\ University of Michigan, Ann Arbor, Mich. 48104, U.S.A.
}

(Received 1 November, 1976)

\begin{abstract}
New observations of the out-of-the ecliptic trajectories of type III solar radio bursts have been obtained from simultaneous direction finding measurements on two independent satellite experiments, IMP- 6 with spin plane in the ecliptic, and RAE-2 with spin plane normal to the ecliptic. Burst exciter trajectories were observed which originated at the active region and then crossed the ecliptic plane at about $0.8 \mathrm{AU}$. We find a considerable large scale north-south component of the interplanetary magnetic field followed by the exciters. The apparent north-south and east-west angular source sizes observed by the two spacecraft are approximately equal, and range from $25^{\circ}$ at $600 \mathrm{kHz}$ to $110^{\circ}$ at $80 \mathrm{kHz}$.
\end{abstract}

\section{Introduction}

Direction finding measurements of type III traveling solar radio bursts by spinning spacecraft are able to determine the trajectories of the fast electron streams which generate the radio emission. Since the electrons follow interplanetary field lines, a visualization of the large scale magnetic field configuration is obtained between the Sun and $1 \mathrm{AU}$. The first radio determination of the spiral structure of the magnetic field in the ecliptic was obtained by this technique using the IMP-6 satellite which had its spin plane in the ecliptic (Fainberg et al., 1972). By simultaneously tracking solar bursts with two satellites spinning in different planes, information on the large scale magnetic field both in and out of the ecliptic may by obtained.

This paper reports on simultaneous burst observations by the Goddard Space Flight Center RAE-2 satellite and the University of Michigan experiment on IMP-6. Similar measurements on other bursts were reported by Baumback et al. (1975) using observations from HAWKEYE-1 and IMP-8. However, the burst trajectories presented here show considerably more north-south structure than was reported in the previous study.

The direction finding used data from two spacecraft, RAE-2 and IMP-6. The instrumentation on RAE- 2 is described by Alexander et al. (1975), and that on IMP-6 by Alvarez et al. (1974). As illustrated in Figure 1, the IMP-6 spin plane

* Present address: Centro de Investigación Astronomia, Apartado 264, Merida, Venezuela. 


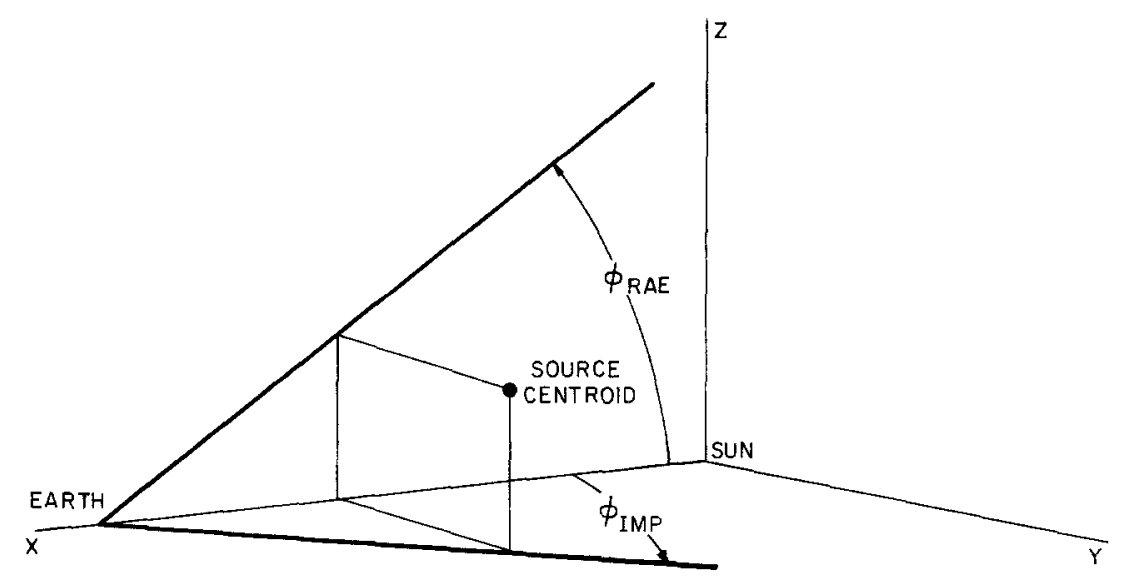

Fig. 1. Diagram showing the components of burst direction measured by IMP-6 with spin plane in the ecliptic (X-Y plane), and by RAE-2 with spin plane normal to the ecliptic (X-Y plane).

was in the ecliptic (X-Y plane) and RAE-2 was spinning in the plane perpendicular to the ecliptic containing the Earth-Sun line ( $\mathrm{X}-\mathrm{Z}$ plane). Each spacecraft utilized a dipole antenna located in its spin plane. Because of satellite spin, the dipole pattern was repeatedly swept across the radio source, resulting in spin modulation of the observed burst profile. From the phase of this modulation, the direction of the source centroid projected in the spin plane was obtained. Details of the direction finding technique and its accuracy are given by Fainberg (1976). As shown in Figure 1, IMP-6 measured the component of source direction in the ecliptic, $\Phi_{\mathrm{IMP}}$, and RAE-2 measured the component out of the ecliptic, $\Phi_{\mathrm{RAE}}$. Together, the two measured angles uniquely determine the direction of the burst.

In addition to burst direction, information on the observed angular extent of the source can be obtained from the depth of spin modulation. With just one spacecraft, there is an ambiguity in the inferred source size because a small source whose centroid is located away from the spin plane can produce the same depth of modulation as a larger source located in the spin plane. By combining data from two satellites spinning in different planes, the source elevation from each spin plane is known, and thus, the apparent source size can also be determined unambiguously.

Two-dimensional direction measurements were made with RAE-2 and IMP-6 during the three-week period beginning June 21,1973, when RAE-2 was placed in a spin-stabilized mode for making observations out of the ecliptic. During this period, about 20 type III bursts were observed which extended to observing frequencies below $80 \mathrm{kHz}$. There was overlapping coverage of data from the RAE-2 and IMP-6 experiments for four of these bursts. The observations of both the exciter trajectory and apparent source size of these events are presented and discussed in this paper. The burst arrival direction data are presented and discussed in Section 2, and the source sizes in Section 3. 


\section{Burst Trajectories}

The arrival directions, $\Phi_{\mathrm{RAE}}$ and $\Phi_{\mathrm{IMP}}$, measured by each spacecraft are listed in Table I for the four bursts. Data are given for frequencies between $80-900 \mathrm{kHz}$, which is the range over which accurate source direction measurements could be made for these bursts. Above $900 \mathrm{kHz}$, the IMP- 6 antenna pattern becomes more complex than that of an electrically short dipole, and below $80 \mathrm{kHz}$ accurate direction measurements were not possible because of the low spin modulation due to the large source region. Since the RAE-2 experiment sampled a larger number of frequencies than the University of Michigan IMP-6 experiment, RAE- 2 data given in Table I were selected to provide overlapping frequency coverage with IMP-6 and to fill in the low frequency part of the observing range.

The burst directions were obtained by fitting the known dipole response function to the observed spin-modulated burst intensity profile. The amplitude

TABLE 1.

Burst arrival directions.

The measured angles are defined in Figure 1

\begin{tabular}{|c|c|c|c|c|}
\hline \multirow{2}{*}{$\begin{array}{l}\text { Frequency } \\
\qquad(\mathrm{kHz})\end{array}$} & \multicolumn{2}{|c|}{$\begin{array}{l}22 \text { June, } 1973^{\mathrm{a}} \\
2013 \text { UT }\end{array}$} & \multicolumn{2}{|c|}{$\begin{array}{l}24 \text { June, } 1973^{\mathrm{a}} \\
0518 \text { UT }\end{array}$} \\
\hline & RAE-2 & IMP-6 & RAE-2 & IMP-6 \\
\hline 900 & & $3 \pm 1^{\circ} \mathrm{W}$ & & $8^{\circ} \mathrm{W}$ \\
\hline 600 & $3 \pm 1^{\circ} \mathrm{S}$ & $4 \pm 1^{\circ} \mathrm{W}$ & $3 \pm 1^{\circ} \mathrm{S}$ & $9 \pm 12^{\circ} \mathrm{W}$ \\
\hline 350 & $1 \pm 1^{\circ} \mathrm{S}$ & $6 \pm 2^{\circ} \mathrm{W}$ & $4 \pm 1^{\circ} \mathrm{S}$ & $14 \pm 1^{\circ} \mathrm{W}$ \\
\hline 230 & $0 \pm 1^{\circ} \mathrm{S}$ & $2 \pm 1^{\circ} \mathrm{W}$ & $4 \pm 1^{\circ} \mathrm{S}$ & $18 \pm 3^{\circ} \mathrm{W}$ \\
\hline 130 & $5 \pm 1^{\circ} \mathrm{S}$ & $3 \pm 2^{\circ} \mathrm{E}$ & $0 \pm 3^{\circ}$ & \\
\hline 110 & $5 \pm 2^{\circ} \mathrm{S}$ & & $10 \pm 4 \cdot \mathrm{N}$ & \\
\hline 96 & $6 \pm 2^{\circ} \mathrm{S}$ & & $10 \pm 6^{\circ} \mathrm{N}$ & \\
\hline 80 & $9 \pm 3^{\circ} \mathrm{S}$ & & $6 \pm 12^{\circ} \mathrm{N}$ & \\
\hline \multirow{2}{*}{$\begin{array}{c}\text { Frequency } \\
(\mathrm{kHz})\end{array}$} & \multicolumn{2}{|c|}{$\begin{array}{l}22 \text { June, } 1973^{\mathrm{b}} \\
0330 \mathrm{UT}\end{array}$} & \multicolumn{2}{|c|}{$\begin{array}{c}22 \text { June, } 1973^{\mathrm{b}} \\
0900 \text { UT }\end{array}$} \\
\hline & RAE-2 & IMP-6 & RAE-2 & IMP_6 \\
\hline 900 & & & & $1 \pm 2^{\circ} \mathrm{E}$ \\
\hline 600 & & & $3 \pm 1^{\circ} \mathrm{N}$ & $6 \pm 2^{\circ} \mathrm{W}$ \\
\hline 350 & $6 \pm 1^{\circ} \mathrm{N}$ & $8^{\circ} \mathrm{W}$ & $6 \pm 1^{\circ} \mathrm{N}$ & $10 \pm 2^{\circ} \mathrm{W}$ \\
\hline 230 & $7 \pm 1^{\circ} \mathrm{N}$ & $13 \pm 4^{\circ} \mathrm{W}$ & $8 \pm 1^{\circ} \mathrm{N}$ & $22 \pm 2^{\circ} \mathrm{W}$ \\
\hline 130 & $7 \pm 2^{\circ} \mathrm{N}$ & $38 \pm 7^{\circ} \mathrm{W}$ & $9 \pm 2^{\circ} \mathrm{N}$ & $51 \pm 5^{\circ} \mathrm{W}$ \\
\hline 110 & $3 \pm 3^{\circ} \mathrm{S}$ & & $3 \pm 5^{\circ} \mathrm{S}$ & \\
\hline 96 & $22 \pm 3^{\circ} \mathrm{S}$ & & $47 \pm 9^{\circ} \mathrm{S}$ & \\
\hline 80 & $54 \pm 9^{\circ} \mathrm{S}$ & $63 \pm 3^{\circ} \mathrm{W}$ & $53 \pm 5^{\circ} \mathrm{S}$ & $68 \pm 5^{\circ} \mathrm{W}$ \\
\hline
\end{tabular}

${ }^{a}$ Bursts coincident with flares from active region, McMath 12390 , located at $\mathrm{S} 12^{\circ}, \mathrm{W} 34^{\circ}$ on June 22 and at $\mathrm{S} 12^{\circ}$, W5 $52^{\circ}$ on June 24.

${ }^{\mathrm{b}}$ No flares observed. Bursts are believed to be associated with the active region, McMath 12379 , which was beyond the limb at $\mathrm{N} 15^{\circ}$, W125 on June 22. 
and the phase and depth of modulation of the response function were adjusted for the best least squares fit to the data near the maximum of the burst profile. The errors given in Table $I$ are those changes in source direction that cause the goodness of fit to degrade by one standard deviation. The accuracy of direction finding depends on the random noise fluctuations in the data, the depth of modulation, the number of data points used, and the presence of modulated background (Fainberg, 1976). The background level was more than $10 \mathrm{~dB}$ below the peak intensity of these bursts and, therefore, had a negligible effect on the measured source directions. The least squares residuals in Table I agree with the random fluctuations in source direction that are predicted due to the bandwidth and integration time of the receivers.

The four bursts were associated with two different active regions. The bursts at 2013 UT on June 22 and 0518 UT on June 24 were associated with flares from the active region McMath 12390, which was located south of the solar equator and west of the central meridian. The burst directions measured both in and out of the ecliptic are consistent with an origin at the active region. The IMP-6 angle measurements are west of the Earth-Sun line on both days and are considerably more westward on June 24 than on June 22, consistent with two days rotation of the Sun. The RAE- 2 angle measurements of the burst at 2013 UT on June 22 are south of the ecliptic, in agreement with the location of the flare site in the southern hemisphere. The RAE-2 measured directions of the burst on June 24 are also south of the ecliptic at the highest frequencies; however, the directions shift north of the ecliptic at lower frequencies, crossing over at $130 \mathrm{kHz}$. Since emission frequency decreases with increasing distance from the Sun, the RAE-2 data show that the June 24 burst exciter crossed the ecliptic plane going south to north as it moved outward from the Sun. The difference in trajectories of these bursts, occurring two days apart but from the same active region, may be due to evolution of the magnetic field configuration, or the exciters may have been injected along different field lines connected to the active region.

The RAE-2 measurements for the other two bursts in Table I, at 0330 UT and 0900 UT on June 22, show that the exciters also crossed the ecliptic, in this case going from north to south. The change in observed position from north to south of the ecliptic is quite large, as seen from $1 \mathrm{AU}$, going from 3-9 degrees north to more than 50 degrees south as the exciter moves out from the Sun. These angular displacements from the ecliptic before and after the crossing are much larger than the uncertainty in the measurements. Neither burst was associated with an observed flare, but the absence of an observable flare association and the very large westward burst angles measured by IMP-6, together, suggest that the initiating flares were at or beyond the west limb. Since the directions of these two June 22 bursts are very similar, they probably originated from the same active region. The associated active region could have been McMath 12379, which was just beyond the west limb and north of the solar equator on June 22, consistent with the RAE- 2 measurements at the highest frequencies for both bursts. 
In order to obtain a three-dimensional picture of the exciter path from the two-dimensional burst arrival directions, it is necessary to adopt a distance scale for the burst emission levels. The burst at 0900 UT on June 22 is shown in the perspective plot of Figure 2. A model was used with the IMP-6 data to obtain the in-ecliptic projection of the trajectory, which was then combined with the RAE-2 measurements to obtain the north-south displacement from the ecliptic plane. The model assumes that the projection of the exciter path on the ecliptic plane is an Archimedean spiral with footpoint at the active region. It is also assumed that the coronal emission levels vary with frequency as $r^{-1.2}$, where $r$ is the distance from the Sun in the ecliptic, and that the emission is generated at twice the local plasma frequency. This is based on stucies of many bursts observed by Fainberg and Stone (1971) with the RAE-1 satellite, and by Alvarez and Haddock (1973) with OGO-5.

The magnetic lines of force inferred from the burst trajectory in Figure 2 leave the Sun from the hemisphere of the active region, bend toward the ecliptic, crossing it at approximately $0.8 \mathrm{AU}$, and continue on to approximately 20 degrees below the ecliptic as seen from the Sun. Accounting for the inclination of the solar equatorial plane to the ecliptic, this corresponds to 15 degrees south heliographic latitude. The trajectory for the burst occurring six hours earlier at $0330 \mathrm{UT}$ is very similar, indicating that the exciters of both bursts followed the same magnetic field configuration. The exciter trajectories for the other two bursts discussed in this paper also bend toward the ecliptic at large solar distances after emerging from an active region in the southern hemisphere. Thus, there is a considerable large scale

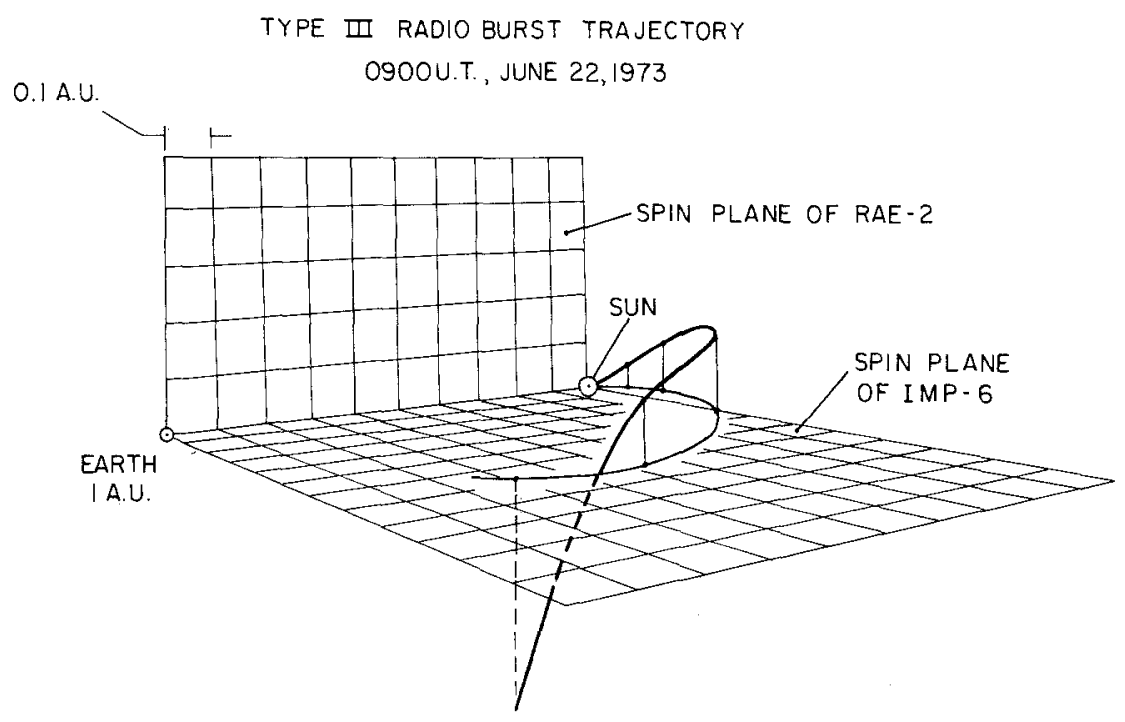

Fig. 2. Burst trajectory obtained from combined RAE-2 and IMP-6 observations, using a model for the distance from the Sun for each emission frequency. The burst exciter follows interplanetary magnetic field lines which cross the ecliptic plane at $0.8 \mathrm{AU}$. At the crossing the field lines are inclined $60^{\circ}$ to the ecliptic. A similar trajectory was observed $5 \frac{1}{2} \mathrm{hr}$ earlier for a burst from the same active region. 
north-south component in the interplanetary magnetic field associated with these active regions.

North-south magnetic field structure was also indicated by the results of Baumback et al. (1975) using observations from 31 to $178 \mathrm{kHz}$ with the spinning spacecraft, HAWKEYE-1 and IMP-8. One of the three burst trajectories which they measured appeared to bend toward the ecliptic after leaving the active region, although it did not cross the ecliptic within $1 \mathrm{AU}$ from the Sun. The other two bursts, which originated from another active region, had trajectories which were approximately radial in their meridional components.

Direct measurements of the solar wind magnetic field have been made only within 7.5 degrees latitude of the solar equatorial plane. Coleman and Rosenberg (1971) have compiled overlapping 27-day means of the north-south component of the field as measured by several spacecraft. During the solar rotation periods studied, they find a systematic skewing of the average field direction such that the magnetic lines of force bend away from the solar equatorial plane, except during several rotation periods of enhanced solar activity, when the average field direction shifted and the lines of force bend toward the equatorial plane. The enhanced activity was indicated by an increase in the amplitude of magnetic field fluctuations. Coleman and Rosenberg suggest that the change in the north-south field component toward the equatorial plane may be due to the increased level of activity.

\section{Size of the Burst Source}

The apparent size of the burst source has been determined from an analysis of the depth of spin modulation of the burst profiles. The analysis assumes that the brightness distribution of the source is gaussian with circular symmetry about the centroid. The source size that is derived from the analysis is the angular width between $e^{-1 / 2}$ points ( $2 \sigma$ width). Previously, source sizes obtained from spin modulation have assumed a uniformly illuminated circular disk as a model distribution. The more realistic gaussian model used here yields source sizes approximately one-half as large as source sizes obtained assuming a uniform disk. If the uniform disk model is used with the data of this paper, then the source diameters agree with previous results (Alvarez, 1976; Baumback et al., 1975).

Since the source elevation from each spin plane is known from the direction measurements, source sizes could be obtained by both spacecraft. The precision in the source size determination is approximately $\pm 3^{\circ}$. Table II shows that the ranges of source sizes measured by each spacecraft at a given frequency are approximately equal. This means that the assumption of circular symmetry of the brightness distribution is valid, since the source diameter is measured in different planes by each satellite.

The data in Table II show that the apparent size of the emitting region is very 
TABLE II

Source diameters.

Range of source diameters as seen from Earth determined from spin modulation of observed bursts. The tabulated values are the $2 \sigma$ widths of the assumed gaussian brightness distributions

\begin{tabular}{cccc}
\hline $\begin{array}{c}\text { Frequency } \\
(\mathrm{kHz})\end{array}$ & $\begin{array}{c}\text { RAE-2 } \\
(\mathrm{deg})\end{array}$ & $\begin{array}{c}\text { IMP-6 } \\
(\mathrm{deg})\end{array}$ & $\begin{array}{c}\text { Mean } \\
(\mathrm{deg})\end{array}$ \\
\hline 600 & $22-28$ & $20-29$ & 25 \\
350 & $22-42$ & $28-38$ & 34 \\
230 & $36-54$ & $38-46$ & 44 \\
130 & $49-72$ & $70-74$ & 66 \\
80 & $92-122$ & $104-120$ & 110 \\
\hline
\end{tabular}

large, in agreement with previous results. Assuming a uniform brightness distribution, Baumback et al. (1975) obtained source sizes as seen from the Sun which were larger than the $60^{\circ}$ extent in solar longitude (between half-maximum flux points) of electron streams associated with type III bursts (Lin, 1974; Alvarez et al., 1975). Even using the gaussian source distribution, we find that the source diameter as seen from the Sun is on the order of 100 degrees. Since this is also much larger than the $\sim 60^{\circ}$ extent expected for the burst exciters, it suggests significant scattering of the burst radiation by irregularities in the solar wind.

\section{Summary}

Direction finding measurements of type III solar radio bursts have revealed large scale north-south structure in the interplanetary magnetic field. The trajectories for two bursts associated with an active region in the northern hemisphere were observed to cross the ecliptic going north to south at a distance of about $0.8 \mathrm{AU}$ from the Sun. One of the two bursts associated with another active region in the southern hemisphere also crossed the ecliptic, in this case going south to north. Thus, the interplanetary magnetic field lines appear to curve away from the latitude of the associated active region (10-15 degrees) and toward the solar equator.

The apparent angular size of the burst source has also been determined. Comparison with the extent in solar longitude of exciter electron streams indicates that the apparent source of the emission has been broadened by coronal ray scattering.

The results show that two-dimensional radio tracking of solar bursts as a function of frequency provides unique information on large scale magnetic field structure out of the ecliptic and over a wide range of solar distances. The use of this technique could be a very valuable tool in future out-of-the-ecliptic studies. 


\section{References}

Alvarez, H.: 1976, Solar Phys. 46, 483.

Alvarez, H. and Haddock, F. T.: 1973, Solar Phys. 29, 197.

Alvarez, H., Haddock, F. T., and Potter, W. H.: 1974, Solar Phys. 34, 413.

Alvarez, H., Lin, R. P., and Bame, S. J.: 1975, Solar Phys. 44, 485.

Alexander, J. K., Kaiser, M. L., Novaco, J. C., Grena, F. R., and Weber, R. R.: 1975, Astron. Astrophys. 40, 365.

Baumback, M. M., Kurth, W. S., and Gurnett, D. A.: 1975, Univ. of Iowa Report 75-45.

Coleman, P. J. and Rosenberg, R. L.: 1971, J. Geophys. Res. 76, 2917.

Fainberg, J.: 1976, NASA/GSFC X-693-76-91.

Fainberg, J. and Stone, R. G.: 1971, Solar Phys. 17, 392.

Fainberg, J., Evans, L. G., and Stone, R. G.: 1972, Science 178, 743.

Lin, R. P.: 1974, Space Sci. Rev. 16, 189. 